\title{
Topical Issue on Non-equilibrium processes in multicomponent and multiphase media
}

\author{
Published online: 12 October 2018 - (c) EDP Sciences / Società Italiana di Fisica / Springer-Verlag GmbH Germany, \\ part of Springer Nature, 2018
}

Most of continuous media participating in natural and technological processes are multicomponent and/or multiphase systems. The physical principles behind the phenomenology of non-equilibrium processes in such media are still not fully understood. To develop a better understanding, a continuous interaction between theory, experiments and numerical simulations is needed. This Topical Issue on non-equilibrium processes in multicomponent and multiphase media aims at contributing to this effort.

The areas of interest for this Topical Issue include hydrodynamic instability and transition to complex non-periodic and chaotic regimes; generation and evolution of large-scale vortex structures in turbulent flows; heat and mass transfer in atmosphere and ocean; hydrodynamics of systems with fluid interfaces; formation of dynamic and dissipative structures at interfaces; thermodiffusion and thermophoresis in molecular fluids and colloidal suspensions; acoustic and wave processes in inhomogeneous media; non-equilibrium processes in complex fluids and in disperse media.

The present Topical Issue also contains papers that were presented at the International Symposium Non-Equilibrium Processes in Continuous Media that was held in Perm (Russia) from May 15 to May 17, 2017.

The current Topical Issue Non-equilibrium processes in multicomponent and multiphase media contains a total of thirteen papers. In the following we provide a brief description of the accepted papers in an order that mirrors the publication dates.

1) In [1] the active flow and dynamics of topological defects in an active nematic interfacial layer confined between immiscible viscous fluid layers is considered. It is found that self-propulsion of positive defects along the direction of their "comet tails" is the principal deterministic component of defect dynamics, while topological and hydrodynamic interactions among mobile defects are responsible for quasi-random jitter.

2) In [2] direct numerical simulations of the gravity-capillary waves induced on a horizontal interface between two slowly miscible liquids is carried out using the phase-field approach. It is found that the interfacial diffusion strongly damps the development of the shorter waves, and, on the other hand, that mixing of the liquids is intensified by the waves, and the role of the waves on the mixing rate is even stronger when the interfacial diffusion is relatively weak.

3) The model of inviscid fluid is widely used for studying different problems in fluid mechanics, but the zero-viscosity limit formally corresponds to an infinite Reynolds number. Consequently, the problem of the existence of the limit of inviscid fluid and its interpretation are continuously attracting attention of physicists and mathematicians. In [3], the role of the scaling laws for the effective turbulent viscosity in turbulent boundary layers is discussed, and these laws are revealed to secure the existence of the limiting passage in case of a turbulent flow.

4) In [4] convection of a binary mixture in a horizontal porous layer under external filtration flux is studied taking into account solute immobilization (solute sorption) within the fractal model of MIM approach. The parametric excitation of convection is observed and the possibility to control convective flow structure by variation of external flux is demonstrated.

5) The terminal orientation of a rigid body in a moving fluid is an example of a dissipative system, out of thermodynamic equilibrium and therefore a perfect testing ground for the validity of the maximum entropy production principle (MaxEP). In [5], the previous analysis is extended to examine i) bodies with fewer symmetries such as a half-ellipse and ii) the system far from thermodynamic equilibrium. Experiments in sedimentation tanks and with hinged bodies in a flow tank support two-dimensional numerical calculations.

6) The dynamic length and time scale separation in suspensions is used to formulate a general description of colloidal thermophoresis in [6]. The suggested approach allows an unambiguous definition of separate contributions to the colloidal flux and clarifies the physical mechanisms behind non-equilibrium motion of colloids. The issuing relations 
for the transport coefficients explicitly show that interfacial thermophoresis has a hydrodynamic character that cannot be explained by purely thermodynamic considerations. Such treatment generalizes the results from other existing approaches, giving them a clear interpretation within the framework of non-equilibrium thermodynamics.

7) In [7] the current state of knowledge of the thermophysical properties of nanofluids is discussed. It is shown experimentally and by molecular dynamics simulation that the viscosity and thermal conductivity of nanofluids generally cannot be described by classical theories for highly dispersed fluids. In particular, their viscosity and thermal conductivity depend not only on the volume concentration of the particles, but also on their size and material.

8) The linear stability of steady Soret-induced convective flow of a ternary mixture placed between differently heated vertical rigid plates is studied in [8]. The stability maps in terms of the Grashof number and net separation ratio are obtained for monotonic and oscillatory longwave and finite-wavelength modes of instability. New finite-wavelength oscillatory instability modes are discovered.

9) The behavior of heavy cylindrical body in a rotating horizontal cylindrical cavity filled with viscous liquid is investigated experimentally in [9]. Several modes of the body behavior depending on the rate of the cavity rotation, i.e., the ratio of the centrifugal force of inertia and the gravity, are detected.

10) Stability of the electro-osmotic flow of electrolyte-dielectric viscous liquids under the influence of the DC and AC electric fields along with the external pressure gradient is studied in [10]. It is found that in the DC case, the external pressure could either stabilize or destabilize the flow depending on the relative directions of the electro-osmotic flow and the pressure-driven flow. In the AC case, as a rule, the external pressure destabilizes the flow.

11) In [11] the canonical Taylor-Green vortex problem is solved by in-house implementation of the novel CABARET numerical scheme in weakly compressible formulation. The features of the numerical method are discussed in terms of the kinetic energy dissipation rate and integral entropy curves, temporal evolution of the spanwise vorticity, energy spectra and spatial correlation functions.

12) The vertical structure of the velocity field induced by solitary waves of the first and second modes in a stratified ideal fluid is studied in [12] in the framework of the weakly nonlinear theory based on the Korteweg-de-Vries-like equation. It is demonstrated that the second-mode soliton is unstable with respect to Kelvin-Helmholtz instability due to large shear on the boundaries between layers.

13) Numerical and experimental investigation of detonation initiation in hydrogen-air mixtures due to focusing of a shock wave reflected inside a wedge are carried out in [13]. The comparison of numerical and experimental results made it possible to validate the developed 3D transient mathematical model: kinetic schemes and turbulence models are improved.

It is a pleasure to acknowledge the participation of all the authors and reviewers for submitting nice papers and reports that allowed the edition of this Topical Issue of The European Physical Journal E. We also wish to warmly thank the journal staff, with a particular thank to Solange Guéhot and to the Editors-in-Chief: François Graner and Francesco Sciortino.

Tatyana Lyubimova, Valentina Shevtsova, and Fabrizio Croccolo Guest Editors

\section{References}

1. Len M. Pismen, Francesc Sagués Eur. Phys. J. E 40, 92 (2017).

2. A. Vorobev, A. Ivantsov, T. Lyubimova Eur. Phys. J. E 40, 99 (2017).

3. Denis S. Goldobin Eur. Phys. J. E 40, 103 (2017).

4. Lyudmila S. Klimenko, Boris S. Maryshev Eur. Phys. J. E 40, 104 (2017).

5. Bong Jae Chung, Blas Ortega, Ashwin VaidyaEur. Phys. J. E 40, 105 (2017).

6. Jérôme Burelbach, Daan Frenkel, Ignacio Pagonabarraga, Erika Eiser Eur. Phys. J. E 41, 7 (2018).

7. Valery Ya. Rudyak, Andrey V. Minakov Eur. Phys. J. E 41, 15 (2018).

8. T. Lyubimova, N. Lobov, V. Shevtsova Eur. Phys. J. E 41, 23 (2018).

9. Olga Vlasova, Nikolai Kozlov Eur. Phys. J. E 41, 26 (2018).

10. E.V. Gorbacheva, G.S. Ganchenko, E.A. Demekhin Eur. Phys. J. E 41, 40 (2018).

11. Yury M. Kulikov, Eduard E. Son Eur. Phys. J. E 41, 41 (2018).

12. Oxana Kurkina, Ekaterina Rouvinskaya, Andrey Kurkin, Ayrat Giniyatullin, Efim Pelinovsky Eur. Phys. J. E 41, 47 (2018).

13. Nickolay N. Smirnov, Oleg G. Penyazkov, Kirill L. Sevrouk, Valeriy F. Nikitin, Lyuben I. Stamov, Veronika V. Tyurenkova Eur. Phys. J. E 41, 66 (2018). 